\title{
Theoretical model of the effect of dimple on friction behavior in hydrodynamic lubrication regime
}

\author{
Auezhan Amanov ${ }^{1,2 *[0000-0002-8695-7430]}$, and Xasan Turkmenov ${ }^{2}$ \\ ${ }^{1}$ Sun Moon University, Asan, Korea \\ ${ }^{2}$ Tashkent Institute of Irrigation and Agricultural Mechanization Engineers, Tashkent, Uzbekistan
}

\begin{abstract}
In this paper, full-film lubrication between the rigid smooth and dimpled surfaces was addressed. A theoretical model is developed to study the effect of a dimple on friction where the smooth surface is rotating while the dimpled surface is at rest. To simplify the problem, the magnified dimple cell is investigated along with some assumptions. Because dimples deploy periodically along with the $x$ and $y$ directions, the lubricant pressure also deploys periodically. A theoretical model can be developed for one cell and then extended to the whole surface. The main goal of this study is to understand the dimple effect on friction in a hydrodynamic lubrication regime. The main applications of this model are several types of bearings (point contact, line contact etc.) and mechanical parts where two surfaces interact in relative motion. Findings the optimum dimensions for the dimples also seem to be one of the interesting research areas in mechanization of agricultural and renewable energy sources.
\end{abstract}

\section{Introduction}

Some researchers have presented the various influences of dimples by investigating the pressure distribution, film thickness, and friction coefficient. Yu and Sadeghi [1] developed a model for a thrust washer containing uniformly distributed radial grooves on the fixed thrust surface. They included the effects of lubricant cavitation in their model. Design curves were generated for load support, friction, and other operating parameters as a function of dimple geometry. They further extended their model by including a thermal effect on thrust washer lubrication. Etsion et al. [2] presented an analytical and experimental study of laser-textured mechanical seals. The friction and temperature were measured and compared with the predictions of a 2D steady-state hydrodynamic model. Their model indicated that the depth-to-diameter ratio was more influential on the pressure generation than the density of dimples. Dimpled thrust surfaces were applied by Vaidya and Sadeghi [3] for orbiting scroll bearings. An analytical model was also developed to compare to the experimental results. They concluded that the friction would decrease when the ratio of the

\footnotetext{
*Corresponding author: avaz2662@sunmoon.ac.kr
} 
dimple depth to film thickness, $h / h_{0}$, or the ratio of the diameter to film thickness, $h / h_{0}$, is large.

For conditions involving full film lubrication, understanding the mechanisms involved in the tribology of dimpled surfaces is mainly derived from investigations of hydrodynamic seals or the simulation of hydrodynamic bearings [4]. Rather few experimental studies have investigated the effects of surface texturing under hydrodynamic lubrication conditions [78]. However, stable fluid films can be obtained between parallel sliding surfaces because of some load support mechanisms, as summarized in Brizmer et al. [9]. Additionally, textured surfaces have been reported to be efficient in modifying lubrication. To date, textured surfaces have been successfully used in many applications, such as thrust bearings, mechanical seals, engine cylinders, etc.

The role of dimples in lubrication, fluid reservoirs, entrapped wear particles, friction and reduced adhesive force has been proven in many experimental studies (Blatter, et al. [10]; Suh, et al. [11]) Ranjan et al. [12]. Experimental and numerical simulations of the effect of dimple size on friction reported by X. Wang [13] concluded that the appropriate dimple size for a specific cylinder radius presents better friction properties. Wan and Xiong [14] proved that a dimpled seal surface could improve the maximum PV value, reduce stiction, trap debris to decrease ploughing, and act as a fluid reservoir. Nanbu, et al. [15] simulated the effect of the geometry of dimples and the relative motion of surfaces on hydrodynamic lubrication performance, which indicated that bottom shapes with wedge and/or step bearings tend to yield thicker films. To improve tribological and lubrication performance of dimpled surfaces, the dimple features should be narrower than the contact width in hydrodynamic lubrication, X. Wang et al. [8-16] and Costa and Hutchings, [17], but wider in a mixed lubrication regime Wakuda et al. [18]. The effect of dimple orientation is important and is involved with load (Costa and Hutchings [17]; Steinhoff et al. [19] and Pettersson and Jacobson [20]). Experimental research reported by Etsion et al. [1], Ronen, et al. [21], and Yu and Sadeghi [1] also observed the influence of dimple depth on the lubricating property and the optimum value.

It is now widely accepted that introducing artificially produced dimples onto sliding surfaces can significantly affect friction, wear, load capacity and pressure distribution of the interacting surfaces in relative motion. The literature review reveals that the majority of the theoretical works have been focused on numerical analyses. Thus, there is a lack of understanding of the nature of the dependence of the interacting parameters with the desired tribological performance measures and various dimple geometries were analyzed. The studies cited above also provide useful insight into dimple dimensions and geometry effects on frictional properties.

In the present paper, various dimple dimensions effects on frictional properties in hydrodynamic lubrication regime are studied theoretically and experimentally. The main objective is to find the ultrasonic nanocrystal surface modification (UNSM) treatment parameters, which for known dimple geometries and given operating conditions provide the best tribological performance. In our theoretical model, the Reynolds equation was used since UNSM-treated dimples shape is hemispherical, and Reynolds is suitable to find a theoretical solution. UNSM technology is a surface modification technology, which produces dimples on the surface [22].

\section{Materials and Methods}

SAE52100 bearing steel with a hardness of 60-62 HRC was used as a sample in this study. The sample's surface was polished using a $\mathrm{SiC}$ sandpaper to achieve a roughness of about $0.10 \sim 0.12 \mu \mathrm{m}$. Dimples were produced on the sample's surface using an ultrasonic nanocrystal surface modification (UNSM) under the parameters listed in Table 1. UNSM 
strikes the sample's surface at a frequency of $20 \mathrm{kHz}$ with a hardened WC ball/tip that results in elastoplastic and surface severe plastic deformation $\left(\mathrm{S}^{2} \mathrm{PD}\right)$, as illustrated in Figure 1. Thus, it produces some dimples on the surface of the sample. Due to a small radius of the ball/tip, the contact area with a sample is relatively small, causing high contact pressure up to $30 \mathrm{GPa}$. Exact dimple dimensions such as diameter and height were measured using an atomic force microscope (AFM).

Table 1. UNSM treatment parameters

\begin{tabular}{|c|c|c|c|c|c|}
\hline Frequency, $\mathrm{kHz}$ & Amplitude, $\mu \mathrm{m}$ & Speed, $\mathrm{mm} / \mathrm{min}$ & Load, $\mathrm{N}$ & Interval, $\mu \mathrm{m}$ & Ball diameter, $\mathrm{mm}$ \\
\hline 20 & 30 & 2000 & 50 & 30 & 2.38 \\
\hline
\end{tabular}

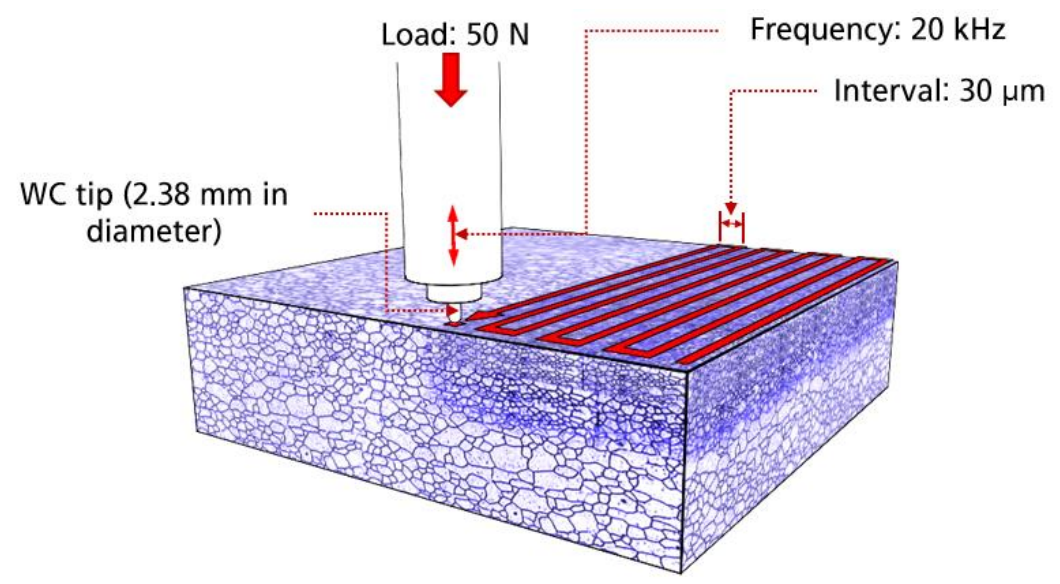

Fig. 1. Schematic view of a UNSM setting

\section{Results and Discussions}

Theoretical Model Considering the Effect of Dimple on Friction

To better understand the effect of dimples on the tribological performance of the parallel surfaces in relative motion and simplify the existing problem, the following assumptions have been made:

1) As the ball is rigid, local elastic deformation is neglected. This assumption implies that no elastohydrodynamic effects are taken into account.

2) The shape of all dimples is identical and hemispherical. The dimples are uniformly distributed over the surface.

3) One column of dimples is representative of the whole surface. Distance between dimples (pitch) is kept the same towards local Cartesian coordinate system $x^{*} y^{*}$.

4) The model is only valid for the hydrodynamic lubrication regime. No asperity contact is allowed.

According to assumption 1, the two parallel surfaces are separated by a uniform oil film of thickness $c$ as shown in Figure 2(a). The assumption of a rigid rotating upper surface allows a first-order approximation of the physical situation without the need for extended numerical solutions. The effects of the curvature of the surface can be neglected in the model. Additionally, since it is in hydrodynamic lubrication regime and therefore requires $c \geq 3 \sigma_{s}$ [23]. Consider, identical hemispherical dimples are uniformly distributed over the lower stationary disk surface, as shown in Figure 2(b). Along the disk specimen surface 
plane, the dimples are circular, and each dimple contained within an imaginary square cell of length $2 r_{i}$, as shown in Figure 2(a).

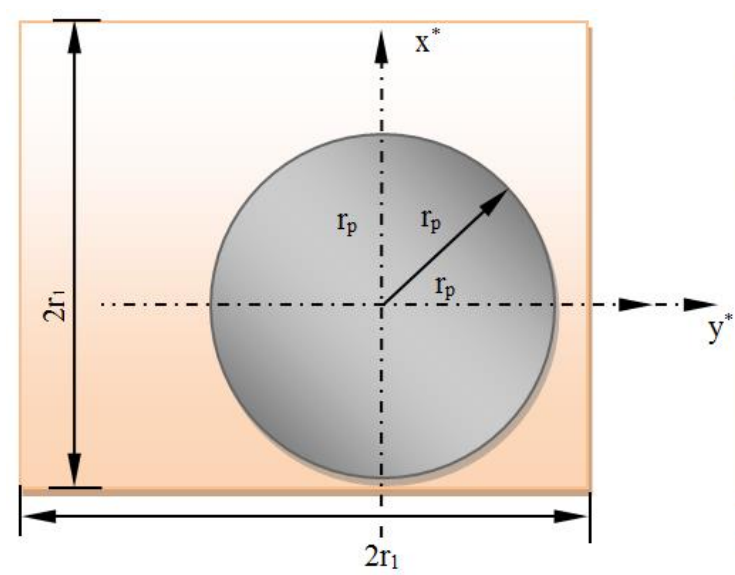

(a)

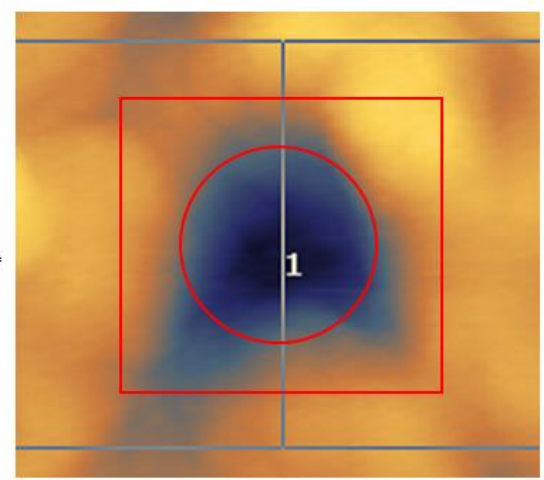

(b)

Fig. 2. Schematic (a) and original (b) single dimple within its imaginary cell (local coordinates).

Figure 2(a) shows one dimple with radius $r_{p}$, positioned at the center of the imaginary cell. We have defined a local Cartesian coordinate system $x^{*} y^{*}$ with origin at the center of the imaginary cell. The imaginary cell half-length $r_{i}$ can be expressed as a function of the dimple density $D_{\text {density }}$, and radius $r_{p}$, respectively as

$$
r_{i}=\frac{r_{p}}{2} \sqrt{\frac{\pi}{D_{\text {density }}}}
$$

Figure 3(a) shows the distribution of the dimples on the disk specimen surface in a global Cartesian $x$ and $y$ coordinate system. Each column of dimples is parallel to the $\mathrm{x}$-axis and can be modeled as shown in Figure 3(b), and each dimple is located at the center of an imaginary cell of size $2 r_{i} \times 2 r_{i}$, as shown in Figure 3(a).

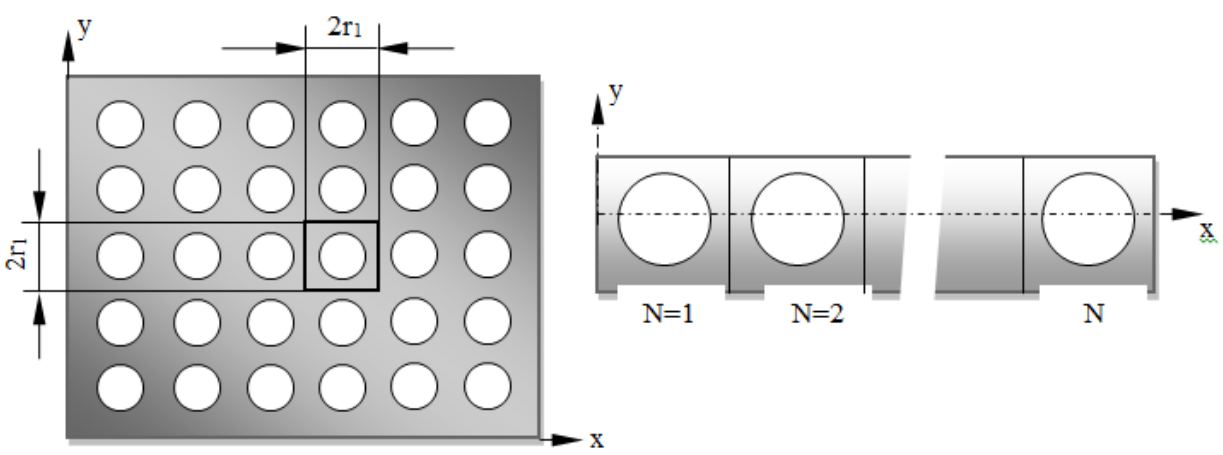

$\mathrm{X}$

Fig. 3. Dimple distribution (a) and a single column of dimple (b).

UNSM-treated surface consists of many dimples. Figure 4 presents a cross through the center of the dimples. Hence, $r_{p}$ denotes the radius of the dimple, $h_{p}$ denotes the depth of the dimple, $c$ is the minimum spacing between two surfaces and $h(x, y)$ is the clearance 
between two parallel surfaces. The distance between two neighboring dimples, $2 r_{i}$, is large enough so that the interaction among the dimples is negligible. $U$ is the linear speed of the rotating upper surface. The dimple density $D_{\text {density }}$ expresses the ratio of the surface covered by dimples versus to total surface area. The hydrodynamic pressure over a single imaginary cell in the film thickness and two dimensional, steady-state incompressible Reynolds equation, which relates the pressure distribution to the spacing at the two surfaces, is given by

$$
\frac{\partial}{\partial x}\left(\frac{h^{3} \partial p}{\eta \partial x}\right)+\frac{\partial}{\partial y}\left(\frac{h^{3} \partial p}{\eta \partial y}\right)=6 \mu U \frac{\partial h}{\partial x}
$$

where: $x$ and $y$ represent coordinates in a global Cartesian coordinate system, $p(x, y)$ is the pressure, $\mu$ is the oil viscosity, and $h(x, y)$ is the local film thickness between two parallel surfaces. Since the two surfaces spacing is much larger than the mean free path, rarefaction effects are neglected.

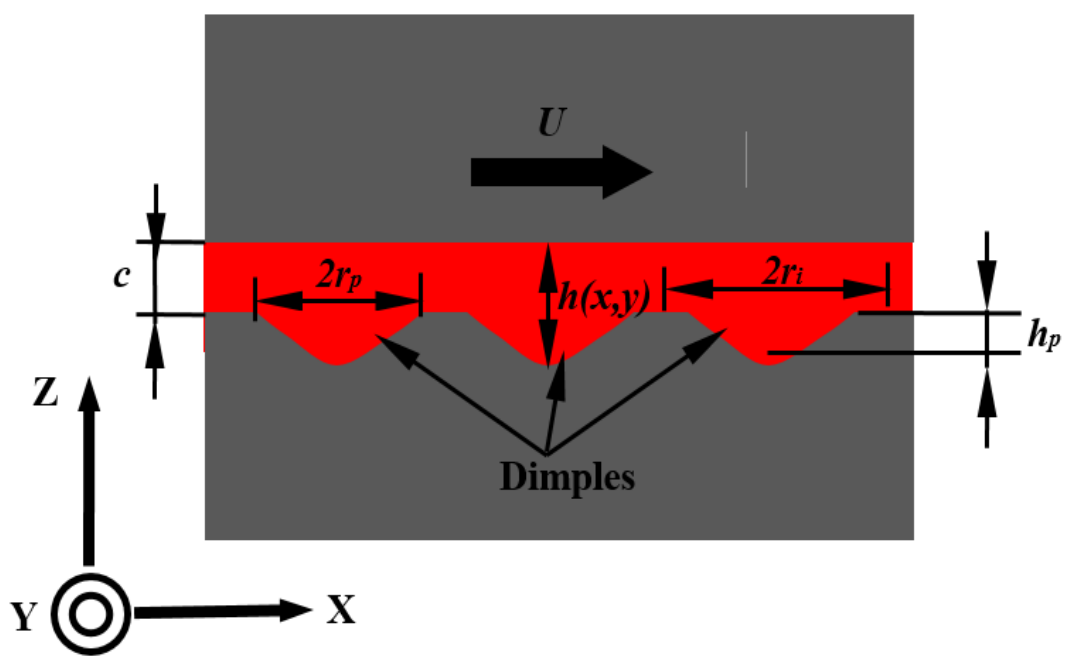

Fig. 4. The geometry of the model.

Outside the dimple, where $x^{2}+y^{2} \geq r_{p}$ in the region $2 r_{i} \times 2 r_{i}$ of an imaginary cell, the local film thickness, $h(x, y)$ is simply equal to the nominal film thickness, $c$. Over the dimples, the local film thickness, $h(x, y)=c+h$, where $h=h_{p}$ at the center dimple; i.e. at $\mathrm{r}=0$. For the remaining area of the dimple, where $x^{2}+y^{2}<r_{p}$, the local film thickness and hence $h$ depends on the profile of the dimple in the $x-y$ plane. The expression of $h$ for hemispherical dimple geometries is shown below:

$$
h^{\prime}=h_{p} \sqrt{1-x^{2}}=r_{p} \sqrt{1-r^{2}}
$$

where: $r$ is the dimple radial coordinate.

Also, in the whole square cell, $h(x, y)$ is the summation of the two parallel spacing $c$ and the dimple depth $h_{p}$ when the point is located in the dimple area, otherwise $h(x, y)$ equal to the two surfaces spacing $c$. It can be expressed in the following form:

$$
\left\{\begin{array}{cc}
c+h_{p}(x, y) \in \text { dimples area } \\
c & \text { else }
\end{array}\right.
$$


In addition to the appropriate expressions for local film thickness, the solution of Equation (3) and to translate Equation (4) into dimensionless form, the following dimensionless coordinates $X$ and $Y$, dimensionless local film thickness $H$ and dimensionless pressure $P$ require the boundary conditions: $\mathrm{P}=0$ at $\mathrm{X}= \pm \mathrm{r}_{\mathrm{i}}$ and $\mathrm{Y}= \pm \mathrm{r}_{\mathrm{i}}$ using the relations

$$
X=\frac{x}{r_{p}}, Y=\frac{y}{r_{p}}, H=\frac{h}{c}, \psi=\frac{h_{p}}{c}, P=\frac{p}{p_{0}}
$$

where $p_{0}$ is the ambient pressure and $\psi$ is the ratio of $h_{p} / c$. The dimensionless film thickness $H$ as a function of the dimensionless local coordinates $X$ and $Y$ for one cell is given by:

$$
H=\left\{\begin{array}{c}
1+\frac{h_{p}}{c}(x, y) \in \text { dimple area } \\
1
\end{array}\right.
$$

When combining Equations (5 and 6), the Reynolds equation in its dimensionless form becomes:

$$
\frac{\partial}{\partial X}\left(H^{3} \frac{\partial P}{\partial X}\right)+\frac{\partial}{\partial Y}\left(H^{3} \frac{\partial P}{\partial Y}\right)=\lambda \frac{\partial H}{\partial X}
$$

where $\lambda=\frac{6 U \mu_{0} r_{p}}{c^{2}}$

Here, outside the dimple boundary, i.e. for $x^{2}+y^{2} \geq 1$, the nondimensional local film thickness, $H_{n}=1$ and the expression for $H_{n}$ over the dimple, where $x^{2}+y^{2}<1$, is shown below for hemispherical profile under consideration.

$$
H_{n}=1+\psi \sqrt{1-R^{2}}
$$

where $R$ is the non-dimensional dimple radial coordinate.

The non-dimensional boundary conditions are $\mathrm{P}=0$ at both $\mathrm{X}= \pm \xi$ and $\mathrm{Y}= \pm \xi$. If the dimple ratio is $\mathrm{S}$; that is, if the dimple area covers $\mathrm{S}$ fraction of the surface area, then for a single imaginary cell of size $2 r_{i} \times 2 r_{i}$, it may be shown that,

$$
\xi=\frac{1}{2}\left(\frac{\pi}{S}\right)^{0.5}
$$

To create a column of dimples of identical shape (see Figure 2(b)), we expand the dimensionless height distribution for a single cell, $H^{*}\left(X^{*}, Y^{*}\right)$, to a column of $N=20$ cells by repeating the height distribution for a single cell for each cell in the column.

The following boundary conditions are assumed. The pressure is atmospheric at the inlet and outlet of the two parallel surface interfaces. In addition, the pressure is periodic in the direction perpendicular to the air flow (Y-direction). This assumption implies that sideflow effects are neglected. Multiple columns of dimples next to one another will yield a periodic pressure distribution. The period is defined by the size of the imaginary cell $\left(2 r_{i}\right)$. The boundary conditions can be expressed as

$$
\begin{gathered}
P(X=0, Y)=1 \\
P\left(X=N \frac{2 r_{i}}{r_{p}}, Y\right)=1
\end{gathered}
$$




$$
\frac{\partial P}{\partial Y}\left(X, Y=-\frac{r_{i}}{r_{p}}\right)=\frac{\partial P}{\partial Y}\left(X, Y=-\frac{r_{i}}{r_{p}}\right)=0
$$

where: $N$ is the number of cells in a column (see Figure 2(b)). Equation (10) can be solved for the pressure distribution if the dimensionless spacing $H(X, Y)$ and the dimensionless parameter $\lambda$ is specified.

The Reynolds Equation (10) was solved by a finite difference method. A grid of $100 \times$ 100 nodes/cell was chosen based on the consideration of both computing time and accuracy. The finite difference method leads to a set of linear algebraic equations for the nodal values of the pressure, which should be solved with the boundary conditions Equation (10). An over-relaxation factor of $\gamma=1.0(\gamma=b / l)$ was used to obtain a faster convergence of the pressure distribution.

Friction Coefficient Model

A useful study on the performance of UNSM-treated surface clearly requires a comparison with a similar smooth untreated surface running under the same conditions. In that case, the validity of the Reynolds equation is assumed. In Cartesian coordinates, it reads

$$
\frac{\partial}{\partial x}\left(\frac{\partial p}{\partial x} h^{3}\right)+\frac{\partial}{\partial y}\left(\frac{\partial p}{\partial y} h^{3}\right)=6 \eta U \frac{\partial h}{\partial x}+12 \eta V
$$

where: $p$ is the pressure, $h$ is the film thickness, $U$ is the tangential relative velocity band, $V$ is the relative normal velocity, and $\eta$ is the viscosity.

The corresponding parameters for the UNSM-treated surface are found from a modified Reynolds equation expressing the effect of the geometry by roughness factors, as originally proposed by Patir and Cheng. ${ }^{[38]}$ This equation may be written as

$$
\frac{\partial}{\partial x}\left(\emptyset_{x} \frac{\partial p}{\partial x} h^{3}\right)+\frac{\partial}{\partial y}\left(\emptyset_{y} \frac{\partial p}{\partial y} h^{3}\right)=6 \eta U \frac{\partial\left(\emptyset_{u} h\right)}{\partial x}+12 \eta V
$$

The chosen dimple geometry consists of a repetition of a unit dimple of dimensions $l$ and $b$ in the $x$ and $y$ directions, respectively. In the present analysis, $b=l$, where $b$ is the UNSM-treated length and $l$ is the dimple column length. The relevant quantities, a load so forth for the UNSM-treated surface are found as described above, solving Equation (19) instead of Equation (18). The dimple shape is shown in Figure 5.

$$
\varepsilon=c\left\{\left[1-\cos \left(\frac{2 \pi x}{l}\right)\right]\left[1-\cos \left(\frac{2 \pi y}{b}\right]-1\right\}\right.
$$

where $\varepsilon$ is the dimple depth, and $c$ is the amplitude for three different dimple sizes, as shown in Figure 6.

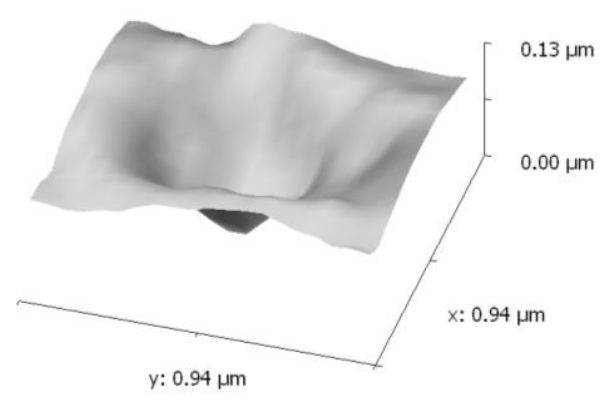

Fig. 5. Unit dimple 


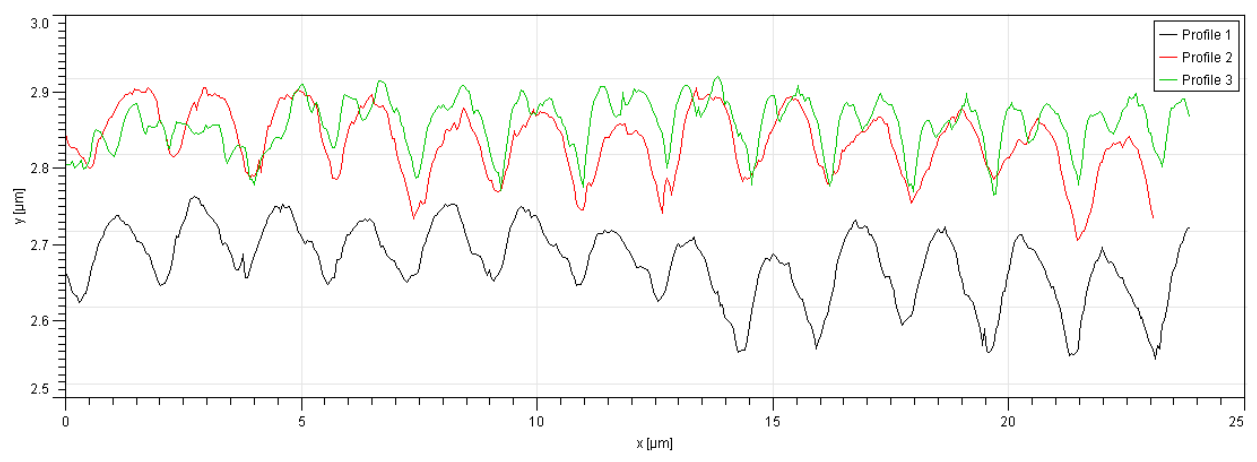

Fig. 6. Amplitude of dimples size.

For polished surface rotating under a hydrodynamic regime, no asperity contact occurs. Hence the friction between two parallel surfaces is entirely due to viscosity. Since we do not include slip boundary regimes, the average shear stress $\tau$ can be approximated as

$$
\tau_{\text {avg }}=\frac{\mu U}{c}
$$

where $\mu$ is the viscosity, $U$ is the upper rotating polished surface speed, and $c$ is the spacing between two parallel surfaces (see Figure 4). An equivalent parallel surfaces spacing $c_{e q}$ should be used in Equation (21), which incorporates the effect of the dimples. This equivalent clearance depends on an equivalent dimple depth that can be obtained by dividing the volume of dimple by the surface of the area of its imaginary square cell, as shown in Figure 2.

$$
h_{e q}=\frac{h_{p} D_{\text {density }}}{6 r_{p}^{2}}\left(h_{p}^{2}+3 r_{p}^{2}\right)
$$

Hence, the equivalent two parallel surfaces spacing can be expressed as

$$
c_{e q}=c+h_{e q}
$$

For the untreated surfaces (smooth) surfaces $h_{e q}=0$ and thus $c_{e q}=c$.

The friction coefficient $f$ of the untreated and UNSM-treated surfaces due to hydrodynamic lubrication can be expressed as

$$
\begin{gathered}
f=\frac{\tau_{\text {avg }}}{p_{\text {avg }}-p_{a}}=\frac{\mu U}{\left(p_{a v g}-p_{a}\right) c} \\
f=\frac{\tau_{a v g}}{p_{a v g}-p_{a}}=\frac{\mu U}{\left(p_{a v g}-p_{a}\right) c_{e q}}
\end{gathered}
$$

where $p_{\text {avg }}$ is the average pressure and $p_{a}$ is the atmospheric pressure.

Since both equivalent spacing $c_{e q}$ and the average pressure $p_{\text {avg }}$ are expected to be higher for the UNSM-treated surfaces than for the untreated surface, the friction coefficient according to Equation 25 will be lower in the hydrodynamic lubrication regime for the UNSM-treated surface than that of the untreated surface. Also, the speed that marks the transition between boundary lubrication and hydrodynamic lubrication regime will be lower in the case of UNSM-treated surface. Indeed, suppose the values of pavg and ceq are higher for UNSM-treated disks. In that case, the speed $U$ to obtain a certain friction coefficient $f$, 
that is associated with the transition from boundary lubrication to hydrodynamic, will be lower.

The theoretical model assumed perfect, symmetric, homogenous and repeatable dimples on the lower stationary surface. In reality, the repeatability of the UNSM technology may not produce homogenously distributed dimples on the surface, and a range of dimple sizes may be produced. Burstein and Ingman [24] employed a statistical method to consider the effects of distributed irregularities in dimple size. Considering a piston-ring like application, the theoretical model was used to consider the effects of a diverse dimple population whose sizes varied according to a normal distribution. Looking at 100 cases with different dimple size distributions, the study concluded that dimple depth and diameter variations have only a small effect on load supported by the dimpled surface. However, the film thickness for the non-identical dimple surface was lower than for the identical dimple case (by a factor of about $2 / 3$, for a $\pm 20 \%$ variation in dimple size), indicating that the expansion of hydrodynamic regime could be smaller for non-uniform dimples.

\section{Conclusions}

In this study, a series of dimples were produced by UNSM technology. The performance of different size of dimpled surfaces on friction coefficient was theoretical model was built. The following conclusions can be drawn based on the results of experimental and analytical tests:

- Produced dimples are strong and can be used to strongly improve the tribological behavior of the targeted (SAE 52100 bearing steel) material under oil-lubricated conditions.

- For all friction performances, the positive dimple size provided the best results compared to the untreated surface.

- These UNSM-treated surfaces can increase lubrication film and fluid velocity and generate pressure between two parallel surfaces.

- More experimental evidence on friction coefficient dependence on dimple size, density is taken into consideration.

To summarize, dimples serve as an oil reservoir to provide lubricant under hydrodynamic lubrication condition. Oil flowing out of the dimple lubricates downstream of the dimple. Increasing the depth of the dimple will extend the lubricant extraction. Increasing the load also extends the lubricant extraction.

\section{References}

1. T.H. Yu, and F. Sadeghi, Groove effects on thrust washer lubrication, J. Trib., 123 (2), pp. 295-304, https://doi.org/10.1115/1.1308014. (2001),

2. I. Etsion, Y. Klingerman, and G. Halperin, Analytical and experimental investigation of laser-textured mechanical seal faces, Tribol. Trans., (42), pp. 511-516, https://doi.org/10.1080/10402009908982248. (1999),

3. A. Vaidya, and F. Sadeghi, Hydrodynamic lubrication of scroll compressor thrust bearing with grooves and circular pockets, ASHRAE Trans., 114 (2), pp. 411-421, (2008).

4. A. Vaidya, and F. Sadeghi, Experimental investigation of orbiting thrust bearing using wide and shallow circular pockets, ASHRAE Trans., 115 (2), pp. 668-675, (2009). 
5. D.B. Hamilton, J.A. Walowit, and C.M. Allen, A theory of lubrication by microasperities, ASME J. Basic Eng. Mater., 88 (1), pp. 177-185. https://doi.org/10.1115/1.3645799. (1966).

6. I. Etsion, and L. Burstein, A model for mechanical seals with regular microsurface structure, Tribol. Trans., 39 (3), pp. 677-683, , https://doi.org/10.1080/10402009608983582. (1996).

7. M. Geiger, S. Roth, and W. Becker, Influence of laser-produced microstructures on the tribological behaviour of ceramics, Surf. Coat. Technol., 100-101, pp.17-22, https://doi.org/10.1016/S0257-8972(97)00581-1. (1998),

8. X. Wang, K. Kato, K. Adachi, and K. Aizawa, The effect of laser texturing of SiC surface on the critical load for the transition of water lubrication mode from hydrodynamic to mixed, Tribol. Int., 34, pp. 703-711, https://doi.org/10.1016/S0301679X(01)00063-9. (2001),

9. V. Brizmer, Y. Kligerman, and I. Etsion, A laser surface textured parallel thrust bearing, Tribol. $\quad$ Trans., $46 \quad$ (3), pp. 397-403, https://doi.org/10.1080/10402000308982643. (2003),

10. A. Blatter, M. Maillat, S.M. Pimenov, G.A. Shafeev, A.V. Simakin, and E.N. Loubnin, Lubricated sliding performance of laser-patterned sapphire, Wear, 232, pp. 226-230, https://doi.org/10.1016/S0043-1648(99)00150-7. (1999),

11. N.P. Suh, M. Mosleh, and P.S. Howard, "Control of friction", Wear, 175, pp. 151158, https://doi.org/10.1016/0043-1648(94)90178-3. (1994),

12. R. Ranjan, D. N. Lambeth, M. Tromel, P. Goglia, and Y. Li, Laser texturing for lowflying-height media, J. Appl. Phys., 69 (8), pp. 5745-5747, https://doi.org/10.1063/1.347908. (1991),

13. X. Wang, W. Liu, F. Zhou, and D. Zhu, Preliminary investigation of the effect of dimple size on friction in line contacts", Trib. Int., 42 (7), pp. 1118-1123, https://doi.org/10.1016/j.triboint.2009.03.012. (2009),

14. Y. Wan, and D.S. Xiong, The effect of laser surface texturing on frictional performance of face seal, J. Mater. Process. Technol., 197 (1-3), pp. 96-100, https://doi.org/10.1016/j.jmatprotec.2007.06.019. (2008),

15. T. Nanbu, N. Ren, Y. Yasuda, D. Zhu, and Q. J. Wang, Micro-textures in concentrated conformal-contact lubrication: effects of texture bottom shape and surface relative motion, Tribol. Lett. 29, pp. 241-252, https://doi.org/10.1007/s11249008-9302-9. (2008),

16. X. Wang, K. Kato, and K. Adachi, The lubrication effect of micro-pits on parallel sliding faces of $\mathrm{SiC}$ in water, Tribol. Trans., 45 (3), pp. 294-301, https://doi.org/10.1080/10402000208982552. (2002),

17. H.L. Costa, and I.M. Hutchings, Hydrodynamic lubrication of textured steel surfaces under reciprocating sliding conditions, Tribol. Int., 40 (8), pp. 1227-1238, https://doi.org/10.1016/j.triboint.2007.01.014. (2007),

18. M. Wakuda, Y. Yamauchi, S. Kanzaki, and Y. Yasuda, Effect of surface texturing on friction reduction between ceramic and steel materials under lubricated sliding contact, Wear, 254, pp.356-363, https://doi.org/10.1016/S0043-1648(03)00004-8. (2003),

19. K. K. Steinhoff, W. Rasp, and O. Pawelski, Development of deterministic-stochastic surface structures to improve the tribological conditions of sheet forming processes, $\mathrm{J}$. Mater. Process. Technol., 60, pp. 355-361, https://doi.org/10.1016/09240136(96)02354-0. (1996), 
20. U. Pettersson, and S. Jacobson, Friction and wear properties of micro textured DLC coated surfaces in boundary lubricated sliding, Tribol. Lett., 17 (3), pp. 553-559, https://doi.org/10.1023/B:TRIL.0000044504.76164.4e. (2004),

21. A. Ronen, I. Etsion, and Y. Kligerman, Friction reducing surface texturing in reciprocating automotive components, Tribol. Trans., 44, pp 359-366, (2001), https://doi.org/10.1080/10402000108982468.

22. M. Arghir, N. Roucou, M. Helene, and J. Frene, Theoretical analysis of the incompressible laminar flow in a macro-roughness cell, J. Tribol. - Trans. ASME, 125 (2), pp. 309-318, https://doi.org/10.1115/1.1506328. (2003),

23. L. Burstein, and D. Ingman, Pore ensemble statistics in application to lubrication under reciprocating motion, Tribol. Trans. 43, pp. 205-212. https://doi.org/10.1080/10402000008982330. (2000),

24. L. Burstein, and D. Ingman, Effect of pore ensemble statistics on load support of mechanical seals with pore-covered faces, J. Tribol. - Trans. ASME, 121(4), pp. 927932, https://doi.org/10.1115/1.2834157. (1999), 